\title{
Culturing of Melanocytes from the Equine Hair Follicle Outer Root Sheath
}

\author{
Hanluo Li $\left.{ }^{1,+}{ }^{(}\right)$, Jule Kristin Michler $\left.{ }^{2,+}{ }^{(}\right)$, Alexander Bartella ${ }^{1}\left(\mathbb{D}\right.$, Anna Katharina Sander ${ }^{1}$, Sebastian Gaus ${ }^{1}$, \\ Sebastian Hahnel ${ }^{3}$, Rüdiger Zimmerer ${ }^{1}$, Jan-Christoph Simon ${ }^{4}$, Vuk Savkovic ${ }^{1, *, \ddagger}$ and Bernd Lethaus ${ }^{1, \ddagger(1)}$
}

Citation: Li, H.; Michler, J.K.;

Bartella, A.; Sander, A.K.; Gaus, S.; Hahnel, S.; Zimmerer, R.; Simon, J.-C.; Savkovic, V.; Lethaus, B. Culturing of Melanocytes from the Equine Hair Follicle Outer Root Sheath. Processes 2021, 9, 177. https://doi.org/ $10.3390 /$ pr9010177

Received: 20 November 2020

Accepted: 13 January 2021

Published: 19 January 2021

Publisher's Note: MDPI stays neutral with regard to jurisdictional claims in published maps and institutional affiliations.

Copyright: (c) 2021 by the authors. Licensee MDPI, Basel, Switzerland. This article is an open access article distributed under the terms and conditions of the Creative Commons Attribution (CC BY) license (https:/ / creativecommons.org/licenses/by/ $4.0 /)$.
1 Department of Cranial Maxillofacial Plastic Surgery, University Hospital Leipzig, 04103 Leipzig, Germany; hanluo.li@medizin.uni-leipzig.de (H.L.); Alexander.Bartella@medizin.uni-leipzig.de (A.B.); anna.sander@medizin.uni-leipzig.de (A.K.S.); Sebastian.Gaus@medizin.uni-leipzig.de (S.G.); Ruediger.Zimmerer@medizin.uni-leipzig.de (R.Z.); Bernd.Lethaus@medizin.uni-leipzig.de (B.L.)

2 Institute of Veterinary Anatomy, University of Leipzig, 04103 Leipzig, Germany; Jule.Michler@vetmed.uni-leipzig.de

3 Polyclinic for Dental Prosthetics and Material Sciences, University Hospital Leipzig, 04103 Leipzig, Germany; Sebastian.Hahnel@medizin.uni-leipzig.de

4 Clinic for Dermatology, Venereology and Allergology, University Hospital Leipzig, 04103 Leipzig, Germany; Jan-Christoph.Simon@medizin.uni-leipzig.de

* Correspondence: Vuk.Savkovic@medizin.uni-leipzig.de; Tel.: +49-341-97-21115

+ The first two authors contributed equally to this work.

$\ddagger$ These authors contributed equally to this work.

\begin{abstract}
Hair follicles harbor a heterogeneous regenerative cell pool and represent a putative lowto-non-invasively available source of stem cells. We previously reported a technology for culturing human melanocytes from the hair follicle outer root sheath (ORS) for autologous pigmentation of tissue engineered skin equivalents. This study translated the ORS technology to horses. We de-veloped a culture of equine melanocytes from the ORS (eMORS) from equine forelock hair follicles cultured by means of an analogue human hair follicle-based in vitro methodology. The procedure was adjusted to equine physiology by addition of equine serum to the culture medium. The hair follicles were isolated by macerating forelock skin rests, enzymatically digested and subjected to airmedium-interface cultivation method. The procedure resulted in differentiated equine melanocytes, which exhibited typical morphology, presence of melanosomes, expression of cytoskeleton proteins vimentin, $\alpha$-SMA, Sox2, S100ß and tyrosinase as well as tyrosinase activity followed by production of melanin. According to all assessed parameters, eMORS could be ranked as partially melanotic melanocytes. The results of the study offer an experimental base for further insight into hair follicle biology in equine and for comparative studies of hair follicles across different species.
\end{abstract}

Keywords: equine hair follicle; outer root sheath; melanocytes; horse

\section{Introduction}

The therapeutic potential of adult stem cells has putatively fueled regenerative medicine research in the several past decades. Initially, the standard methods of obtaining adult stem cells have been relying on invasive, biopsy-based approaches, which involved substantial donor site morbidity. More recently, the alternative, less invasive methods of harvesting stem cells have been progressively gaining momentum. To this day, the hair follicle has remained the least invasively obtainable source of stem cells as well as the one with the most favorable sample/yield ratio.

Initially, stem cells residing in the outer root sheath (ORS) of the hair follicle were identified in the bulge area adjacent to the sebaceous gland [1]. ORS stem cell pluripotency and their potential in regenerative medicine were shown in subsequent studies [2,3]. In 1995, the first successful long-term cultivation of proliferating hair follicle melanocytes was described, sparking the hope that they might be a key for hair regrowth in alopecia areata 
and for repigmentation of the epidermis in vitiligo, burns and wounds [4]. An array of routine applications for culturing skin cells from the ORS followed, designated for wound healing and for treatments of chronic depigmentation disorders, e.g., vitiligo [5-14].

All these procedures required a safe, low-to-non-invasive method of obtaining follicles in the first place, as well as a reliable, reproducible culture of single or multiple cell types from the obtained follicles with a high-end yield. In pursuit of this goal, a method of culturing keratinocytes from plucked follicle ORS was postulated by Hunziker in 2003 [9], followed by an improved technology for culturing human melanocytes based on it [14-16]. Here, a relatively simple air-liquid-interface experimental set-up helped taking advantage of the migratory capability of the ORS cells, enabling a smooth transfer onto the nylon mesh of a suspended well. The stem cells and skin cell precursors proliferated and differentiated in the further course of culture, resulting in pure single cultures of keratinocytes and melanocytes. Challenge of selecting melanocytes was solved by standardized selection techniques such as withdrawal of the proximal, fibroblast-rich follicle part, differential trypsinization of easily detachable melanocytes and Geneticin impact on quickly dividing keratinocytes and fibroblasts. The cultured melanocytes were named human melanocytes from the outer root sheath of hair follicle (HUMORS) [15].

This study presents a re-visited method for obtaining HUMORS applied to horse forelock hair follicles in order to culture equine melanocytes from the outer root sheath (short: eMORS). We based the study design on the anatomic resemblance between human and equine long hair follicles, which led to a hypothesis that an analogue technical procedure in horse hair follicles could yield a pure and putative equine melanocyte culture as well, if equine physiological conditions were mimicked in vitro.

With the technology ready to be translated, we aimed at providing characteristics of equine hair follicle melanocyte cultures in order to allow further investigations of this subpopulation in horses. To this day, no studies on equine melanocytes in vitro have been made available. Hereby, ground would be gained for comparative studies of hair follicles and placode structures across different species, in a good hope of analogously culturing other cell types from hair follicles of multiple species for purposes of regenerative applications.

\section{Materials and Methods}

Intact hair follicles were obtained by dissecting surgically removed forelock skin rests from the superior frontal region $(\mathrm{N}=30)$ available as waste material from slaughter horses at a local abattoir and from experimental animals that were euthanized for reasons unrelated to this study. None of the animals had clinical signs of a skin disease. All experiments involving human samples were institutionally approved (427/16-ek) by the Ethical Committee of Medical Faculty, University of Leipzig.

Full-thickness skin part sized approximately $9 \mathrm{~cm}^{2}$ was excised $30 \mathrm{~min}$ post mortem and transferred to washing solution (PBS containing Penicillin/Streptomycin $(100 \mathrm{U} / \mathrm{mL}$ ) and Amphothericin B $(82.5 \mu \mathrm{g} / \mathrm{mL})$, both from ThermoFisher Scientific, Darmstadt, Germany).

For purposes of melanocyte characterization, equine skin sections were used as controls for tyrosinase protein expression and distribution and Fontana Masson staining, whereas Normal Human Epidermal Melanocytes (NHEM, Promocell GmbH, Heidelberg, Germany) were used as controls for assessment of melanin content and L-DOPA conversion. Ex vivo experiments of isolating follicles from equine skin and culturing melanocytes according to the procedure presented herein were performed 28 times $(\mathrm{N}=28)$. In vitro assessments were performed on material of at least 3 horses in 3 experimental replicates.

\subsection{Skin Sectioning for Histological Staining}

Horse forehead skin was dissected, washed in PBS, fixed in 4\% PFA overnight, dehydrated, embedded in paraffin and sectioned in thickness of $2 \mu \mathrm{m}$. After deparaffinization and rehydration, a standard Hematoxylin-Eosin staining (Hematoxylin-Eosin, Carl Roth $\mathrm{GmbH}$, Karlsruhe, Germany) was carried out. Additionally, Fontana Masson staining was performed as a special stain showing the argentaffin melanocytes. For this, the sections 
were incubated in ammoniac silver solution (Carl Roth GmbH, Karlsruhe, Germany) at $60{ }^{\circ} \mathrm{C}$ for $60 \mathrm{~min}$. After rinsing, the sections were incubated in Gold Chloride Solution $(0.2 \%$, Carl Roth $\mathrm{GmbH}$, Karlsruhe, Germany) for $10 \mathrm{~min}$, and sequentially in Sodium Thiosulfate Solution (5\%, Carl Roth GmbH, Karlsruhe, Germany) for 2 min. The nuclei were counterstained using hematoxylin (Carl Roth $\mathrm{GmbH}$, Karlsruhe, Germany), and mounted with ROTI ${ }^{\circledR}$ Mount (Carl Roth $\mathrm{GmbH}$, Karlsruhe, Germany).

In order to assess the number of horse hair follicles per $\mathrm{cm}^{2}$ of skin, microscopic sections (layer thickness $5 \mu \mathrm{m}$ ) were produced from skin samples. Using five randomly selected areas per specimen, the number of follicles on a surface of $0.5 \mathrm{~cm}^{2}$ was determined manually.

Stained sections were imaged using Keyence BZ-9000 microscope (Keyence GmbH, Neu-Isenburg, Germany) or Nikon TE2000S (Nikon GmbH, Düsseldorf, Germany).

\subsection{Isolation of Forelock Hair Follicles}

The skin was repeatedly rinsed with washing solution. After washing, skin was enzymatically treated by Collagenase V for $10 \mathrm{~min}$. Subsequently, the epidermis was peeled apart from the dermis aided by scalpel and forceps, leaving proximal parts of the hair follicle exposed. The protruded follicle base was trapped by fine forceps and gently pulled in direction of the dermis until full extraction.

\subsection{Processing of the Dissected Hair Follicles}

The follicles were assembled in a cell strainer and washed five times for $1 \mathrm{~min}$ in DMEM supplemented with $100 \mathrm{U} / \mathrm{mL}$ Penicillin/Streptomycin and $2.5 \mu \mathrm{g} / \mathrm{mL}$ Amphotericin B. Each new round of washing was performed by placing the strainer in a separate 6-well and submerging it in a fresh stock of washing solution. Subsequently, the follicles were washed in a well with PBS.

The isolated follicles were shortened at the base in order to eliminate the fibroblast-rich proximal part, which takes approximately one-fifth of the follicle length [16]. The truncated follicles were further enzymatically treated by Collagenase $V$ for $10 \mathrm{~min}$ in order to partially digest main collagen components of the extracellular matrix (ECM). The enzymatically processed follicles were washed by submerging them into a Petri dish with PBS.

\subsection{Primary Air-Liquid-Interface Culture}

Washed follicles were placed on the nylon mesh of a 12-well $(24 \mathrm{~mm})$ Transwell ${ }^{\circledR}$ suspended wells (Corning Inc., New York, NY, USA) in groups of 10 per Transwell. The medium was added from the lower side of the mesh until it made contact with the mesh itself. Medium was further distributed by surface tension forces, covering the follicles with a thin wet film sufficient to supply the nutrients to the cells and to protect the follicles from desiccation, simultaneously permitting the necessary diffusion of the hypoxic gas mixture to the follicles. The formulation of the medium used for culture is an adapted formulation of the DermaLife Melanocyte Medium (DLM, DermaLife, Lifeline Cell Technology, Frederick, MD, USA) enriched by 10\% horse serum and $100 \mathrm{U} / \mathrm{mL}$ Penicillin/Streptomycin $/ 2.5 \mu \mathrm{g} / \mathrm{mL}$ Amphotericin (in further text DLMS). The culture with DLM medium without equine serum was run as control $(\mathrm{N}=3)$. Medium formulation of DLMS is shown in Table 1.

The air-liquid interface primary culture was incubated in hypoxic conditions $\left(5 \% \mathrm{O}_{2}\right.$, $5 \% \mathrm{CO}_{2}$ and $90 \% \mathrm{~N}_{2}$ ) at $37^{\circ} \mathrm{C}$. Medium was changed every 3 days. The cultures were photo-documented once a week by the means of phase-contrast microscope (Nikon Eclipse TS100, Nikon GmbH, Düsseldorf, Germany)

At the point of $40 \%$ confluence on the nylon mesh, culture medium was added until the cells were fully submerged. Primary culture was maintained for 2 to 4 weeks until $90 \%$ confluence on the nylon mesh was reached. 
Table 1. Formulation of the melanocyte culture medium.

\begin{tabular}{cc}
\hline DLMS & Concentration \\
\hline Basal Medium & $88 \%$ \\
\hline L-Glutamine & $6 \mathrm{mM}$ \\
\hline Calcium Chloride & $0.2 \mathrm{mM}$ \\
\hline Epinephrine & $1 \mu \mathrm{M}$ \\
\hline Vitamin C & $50 \mu \mathrm{g} / \mathrm{mL}$ \\
\hline Insulin & $5 \mu \mathrm{g} / \mathrm{mL}$ \\
\hline & $1 \%$ \\
StiMel8 Supplements & Containing: FBS, rhEGF, rhFGF-b, \\
& Endothelin-1, \\
& Apo-Transferrin, Bovine Pituitary Extract \\
Hendrocortisone Hemisuccinate
\end{tabular}

\subsection{Melanocyte Culture}

At $90 \%$ confluence, the cells were detached from the mesh by $0.05 \%$ Trypsin/EDTA, labelled as passage zero (P0), transferred to adherent culture in polystyrene 6-wells, cultured for another 2 weeks and subcultured at $90 \%$ confluence. The culture conditions of this set-up preferentially supported melanocyte culture. Nevertheless, the culture at this stage was still an assembly of melanocyte precursors, melanocytes, keratinocytes and fibroblasts in varying amounts and obtaining a pure melanocyte culture required additional selection procedures, presented in further text $[15,16]$.

At each passage, differential trypsinization was performed under microscope-assisted visual control (approximately for $3 \mathrm{~min}$ ) using Trypsin/EDTA, hereby detaching the habitually weakly adherent melanocytes. The early released cell fraction was collected and passaged further.

Additionally, Geneticin-based selection was performed by applying $50 \mu \mathrm{g} / \mathrm{mL}$ Geneticin (Gibco, ThermoFisher Scientific Inc., Waltham, MA, USA) for $48 \mathrm{~h}$ in order to eliminate quickly dividing keratinocytes and fibroblasts that were dependent on intensive protein synthesis [15-17]. The slowly dividing melanocytes were not strongly impacted by the Geneticin treatment. After $24 \mathrm{~h}$, medium with Geneticin was removed and fresh culture medium was replenished for further culture. The eMORS cell culture was maintained over 12 passages.

For cell doubling time, the cells were trypsinized at $80 \%$ confluence, detached, suspended and subcultured at a 1:5 ratio. At each passage, the cells were counted by the means of hemocytometer and Trypan Blue staining to rule out the necrotic cells. Cell doubling time was calculated based on previously described method by Korzynska et al. [18].

\subsection{L-DOPA Conversion Test}

The eMORS in P8 were detached by trypsin/EDTA, washed in PBS and lysed in 3 freeze/thaw cycles. The disrupted cell content was centrifuged and the pellet was separated from the supernatant. Volume of $100 \mu \mathrm{L}$ of eMORS cell lysate was incubated in $200 \mu \mathrm{L}$ $5 \mathrm{mM}$ L-DOPA solution (in $0.1 \mathrm{M} \mathrm{KH}_{2} \mathrm{PO}_{4}$ buffer, $\mathrm{pH} 7.2$ ) for $4-5 \mathrm{~h}$ at $37^{\circ} \mathrm{C}$ in the dark. The absorbance of the supernatant was measured by the means of spectrophotometry (Synerge, BioTek Instruments Inc., Winooski, VT, USA) at $475 \mathrm{~nm}$ measure wavelength/620 nm reference wavelength and calibrated by a $0.1 \mathrm{M} \mathrm{KH}_{2} \mathrm{PO}_{4}$ buffer blank control. Readout of each sample was compared to that of a synthetic melanin curve based on a $0-100 \mu \mathrm{g} / \mathrm{mL}$ concentration range (Sigma-Aldrich $\mathrm{GmbH}$, Schnelldorf, Germany), retrieving melanin 
concentration of the measured sample by linear regression. The output presented the amount of melanin per ml of lysate. NHEM lysates were used as control.

\subsection{Melanin Content}

After lysis and separation of the eMORS pellet from the supernatant, the pellet was incubated in $1 \mathrm{~N} \mathrm{NaOH}$ at $60^{\circ} \mathrm{C}$ for $3 \mathrm{~h}$ with agitation followed by centrifugation. The absorbance of the collected supernatant was measured by a spectrophotometer (Synerge, BioTek Instruments Inc., Winooski, VT, USA) at $475 \mathrm{~nm}$ measure wavelength $/ 620 \mathrm{~nm}$ reference wavelength. The output displayed the amount of melanin per $\mathrm{ml}$ of pellet lysate. Lysate of the NHEM pellet served as control.

\subsection{Characterization of Melanocytes and Expression of Marker Proteins}

The size of the nucleus, the form of soma, the presence of dendrites and of pigmented melanosomes were descriptively assessed in eMORS by the means of microscopy.

Expression of $\alpha$-SMA, Vimentin, Sox2, S100ß and Tyrosinase was assessed by the means of immunofluorescence [19]. $\alpha$-SMA and Vimentin were selected to label the pattern of cytoskeleton [20]. Sox 2 is known as an activator of microphthalmia-induced transcription factor [21]. MITF, a trigger protein of the melanocytic program [22], whereas tyrosinase is the crucial enzyme for melanin synthesis [23].

Primary antibodies against human proteins were used to label the equine $\alpha$-SMA, Vimentin, Sox2, Tyrosinase and S100, relying on high evolutionary conservation of their sequences (https:/ /blast.ncbi.nlm.nih.gov/Blast, Supplemental Table S1). The primary and secondary antibodies used for immunofluorescence are listed in Table 2.

Table 2. List of antibodies used to detect $\alpha$-SMA, vimentin, Sox2, tyrosinase and S100 $\beta$ in horse cells.

\begin{tabular}{|c|c|c|c|}
\hline Protein & Primary Antibody & Secondary Antibody & Known Cross Reactivity in Horse \\
\hline$\alpha$-SMA & $\begin{array}{l}\text { mouse anti-human } \alpha \text { SMA, } \\
\text { mIgG1, Clone 1A4, Abcam Plc, } \\
\text { ab5694 } \\
\text { working dilution 1:100 }\end{array}$ & $\begin{array}{c}\text { goat anti mouse Dylight488, } \\
\text { Biomol GmbH, Hamburg } \\
\text { cat.no: A90-516D2 } \\
\text { 1:500 in PBS }\end{array}$ & - \\
\hline Vimentin & $\begin{array}{c}\text { mouse monoclonal } \\
\text { anti-human vimentin, C9080, } \\
\text { Cy3-conjugated, Sigma } \\
\text { Aldrich } \\
\text { working dilution 1:500 }\end{array}$ & Does not apply & + \\
\hline Sox2 & $\begin{array}{c}\text { rabbit polyclonal anti-human } \\
\text { Sox2, SAB2701974, Sigma } \\
\text { Aldrich } \\
\text { working dilution 1:100 }\end{array}$ & $\begin{array}{l}\text { goat anti-rabbit PE, } \\
\text { Sigma-Aldrich, } \\
\text { cat.no: P9537 } \\
\text { 1:200 in PBS }\end{array}$ & - \\
\hline Tyrosinase & $\begin{array}{c}\text { mouse monoclonal } \\
\text { anti-human tyrosinase, } \\
\text { MFCD01322752, Sigma } \\
\text { Aldrich }\end{array}$ & $\begin{array}{c}\text { goat anti mouse Dylight } 488, \\
\text { Biomol GmbH, Hamburg } \\
\text { cat.no: A90-516D2 } \\
\text { 1:500 in PBS }\end{array}$ & - \\
\hline S100ß & $\begin{array}{c}\text { working dilution 1:100 } \\
\text { mouse monoclonal anti- } \\
\text { mouse S100ß, 612376, BD } \\
\text { Transduction Laboratories } \\
\text { working dilution 1:50 }\end{array}$ & $\begin{array}{c}\text { goat anti mouse Alexa Fluor } \\
\text { 488, Jackson ImmunoResearch } \\
\text { via Dianova GmbH, Hamburg } \\
\text { cat.no: } 115-545-062 \\
\text { 1:500 in PBS }\end{array}$ & - \\
\hline
\end{tabular}

Briefly, subconfluent cell cultures seeded on 48 well chambers or chamber slides were fixed with $4 \%$ buffered paraformaldehyde for $10 \mathrm{~min}$, washed twice with PBS, and either stained directly or stored at $4{ }^{\circ} \mathrm{C}$. For blocking and permeabilizing, Triton X100 $(0.25 \%$ in PBS) with $5 \%$ normal serum (depending on the species the secondary antibody was 
raised in) was applied for $30 \mathrm{~min}$. The primary antibody was either administered overnight $\left(4{ }^{\circ} \mathrm{C}\right)$ or at room temperature for $4 \mathrm{~h}$. After washing, the secondary antibody and the nuclear counterstain (Hoechst 33342, ThermoFisher Scientific Inc., Waltham, MA, USA) was applied for $2 \mathrm{~h}$ at room temperature in the dark.

\subsection{Characterization of Melanocytes and Expression of Marker Genes}

To determine the melanocytic gene expression in equine melanocytes at the early and late passages, qRT-PCR was used. Briefly, eMORS at P2 and P10 were detached using trypsin/EDTA, lysed in Qiazol Lysis Reagent (Qiagen, Hilden, Germany) and total RNA was isolated using RNeasy Plus Universal Kit (Qiagen, Hilden, Germany). The mRNA was reversely transcribed to cDNA by QuantiTect Reverse Transcription Kit (Qiagen, Hilden, Germany), and qRT-PCR was performed in a 7500 Real-Time PCR System (ThermoFisher Scientific, Darmstadt, Germany) using QuantiFast SYBR ${ }^{\circledR}$ Green PCR Kit (Qiagen, Hilden, Germany). PCR cycling was set at $95^{\circ} \mathrm{C}$ for $60 \mathrm{~s}$, followed by 40 cycles of $95^{\circ} \mathrm{C}$ for $10 \mathrm{~s}$, and $60{ }^{\circ} \mathrm{C}$ for $30 \mathrm{~s}$. Gene expressions were normalized to the housekeeping gene hypoxanthineguanine phosphoribosyltransferase-1 (HPRT-1), and analyzed using $2^{-\triangle \mathrm{Ct}}$ method. Primer sequences for the horse melanocyte genes are specified in Table 3.

Table 3. Primer sequences used to detect MITF, TYR, CKIT and PMEL in eMORS.

\begin{tabular}{|c|c|}
\hline Gene & Primer Sequence \\
\hline MITF for & CAAGGCAGGATCCATCAA \\
\hline MITF rev & TGACATCATTAGCCTAGAATCAAGT \\
\hline$T Y R$ for & CAGATCCСTGTACCTAGAAGAT \\
\hline$T Y R$ rev & TTTCGAAGCAGTATGCACAA \\
\hline CKIT for & ATCAGCGCATAACAGCCTAAT \\
\hline CKIT rev & CCAGCAAAATCAGAGTTAATCG \\
\hline$P M E L$ for & ACTCTTTGACTCCTCACACAGC \\
\hline$P M E L$ rev & ATTTCAAATGGGGATCATAATGT \\
\hline HPRT-1 for & СТTTТССАААТССТСАGСАTAAT \\
\hline HPRT-1 rev & AGGTCTTGTTCTGATCCTTCTGT \\
\hline
\end{tabular}

\subsection{Statistical Analysis}

Statistical analysis on the quantitative data was performed by an unpaired student's $t$-test. The $p$ values $\leq 0.05$ were regarded as statistically significant.

\section{Results}

\subsection{Staining of Histological Sections from Equine Skin}

Hair follicles in equine skin sections were observed microscopically using standard H\&E staining (Figure 1A). As comparable to human scalp skin, primary hair follicles were evenly distributed. In average, 521 hair follicles were counted per $\mathrm{cm}^{2}$ in the equine skin sections (Figure 1B).

Fontana Masson staining revealed melanin-rich point aggregations that fit the habitual form and distribution of melanosomes in the basal layer of the epidermis and at the periphery of the hair follicle ORS surrounded by dermis (Figure 1C). Tyrosinase fluorescent signals were situated throughout the epidermal basal layer (Figure 1D) and in particular at the mid and proximal portion of the hair follicle ORS (Figure 1E). 


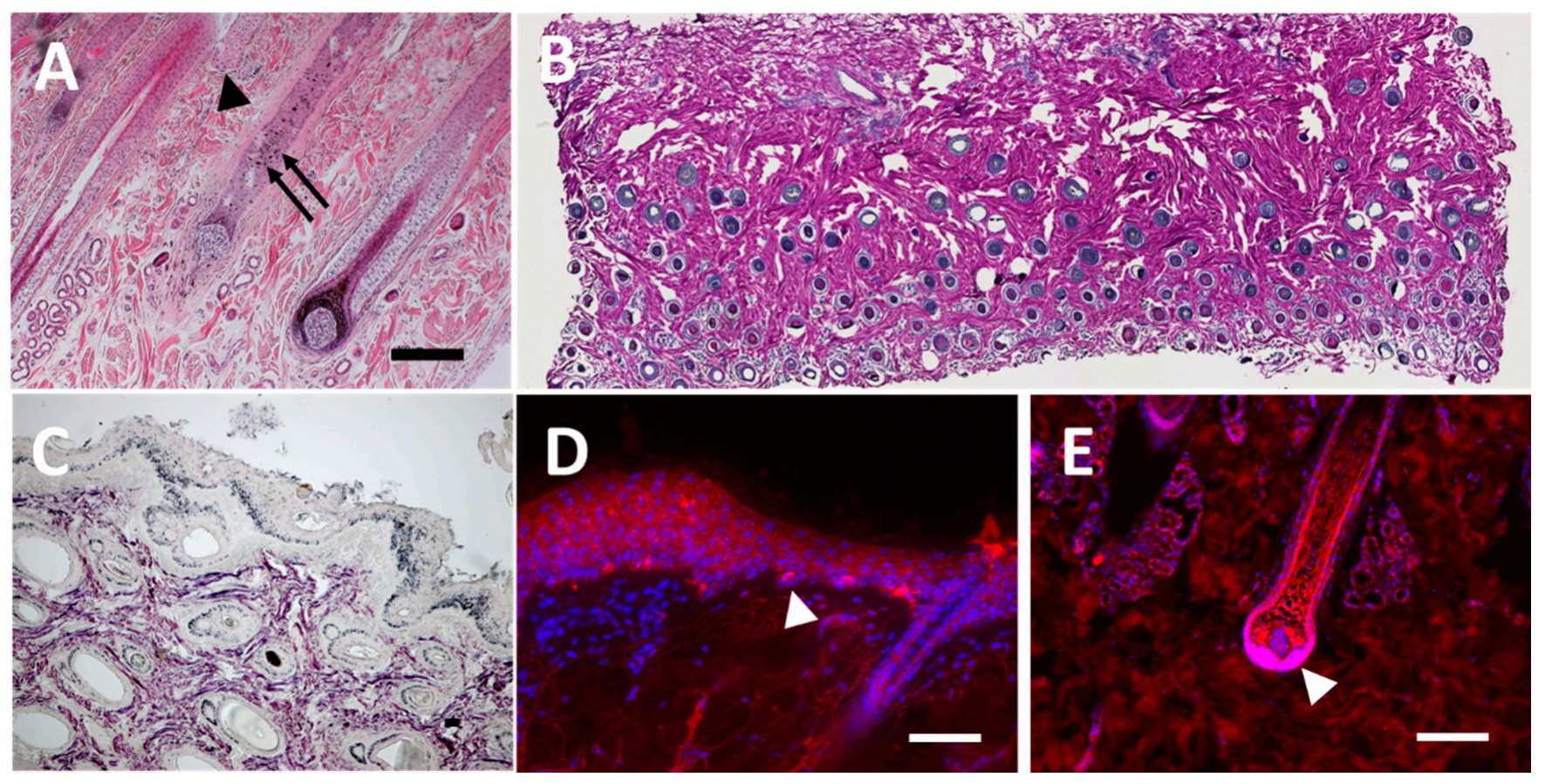

Figure 1. H\&E, Fontana Masson stain and tyrosinase immunofluorescent labeling of horse forehead skin. (A) Histological cross-section of the equine forehead skin was stained with H\&E for morphological analysis. The hair follicles' base is surrounded by sweat glands (arrowhead, A). Outer root sheath (ORS) melanocytes showing melanin deposits are marked with arrows (arrows, A). (B) Perpendicular section of horse forehead skin with observable hair follicles. (C) Fontana Masson-staining of metallic silver deposit in melanocytes in both hair follicle ORS and the basal layer of the epidermis. $(D, E)$ Immunostaining of tyrosinase in horse forehead skin specimen displayed positive staining of functional melanocytes in the basal layer of the epidermis (arrowhead, D) as well as that of the proximal bulb and matrix region (arrowhead, E). Scale bar $100 \mu \mathrm{m}$. (A,D,E) 40× magnification; (C) $4 \times$ magnification; (B): composite image at $4 \times$ magnification.

\section{2. eMORS Culture Parameters}

The cells cultured in DLMS migrated from the ORS of equine hair follicles and populated the nylon mesh of Transwell inserts in $51 \pm 11 \%$ of processed follicles after 7 days. The outgrowth efficiency increased after 14 days, with $62 \pm 11 \%$ follicles yielding migrating cells that populated nylon mesh $\left({ }^{*} p=0.022\right.$ ) (Figure $2 \mathrm{~A}-\mathrm{C}$ ). Interindividual differences concerning outgrowth capacity/velocity were apparent.

Air-liquid interface cultures yielded a viable adherent eMORS cell culture in polypropylene cell culture dishes (Figure 2D). The primary culture of eMORS exhibited a doubling time of $2.07 \pm 0.76$ days. The cells were cultured until passage 12 without having observed major morphological signs of senescence. Control set-ups in DLM without equine serum resulted in migration at a non-progressive rate (data not shown) and failed to reach subcultivation phase in cell culture flasks. The eMORS cells in culture assumed morphology of differentiated melanocytes, with a large nucleus and tri- to polydendritic soma. In perinuclear space and soma, dark pigment accumulations in melanosome-like vesicles were observed. 


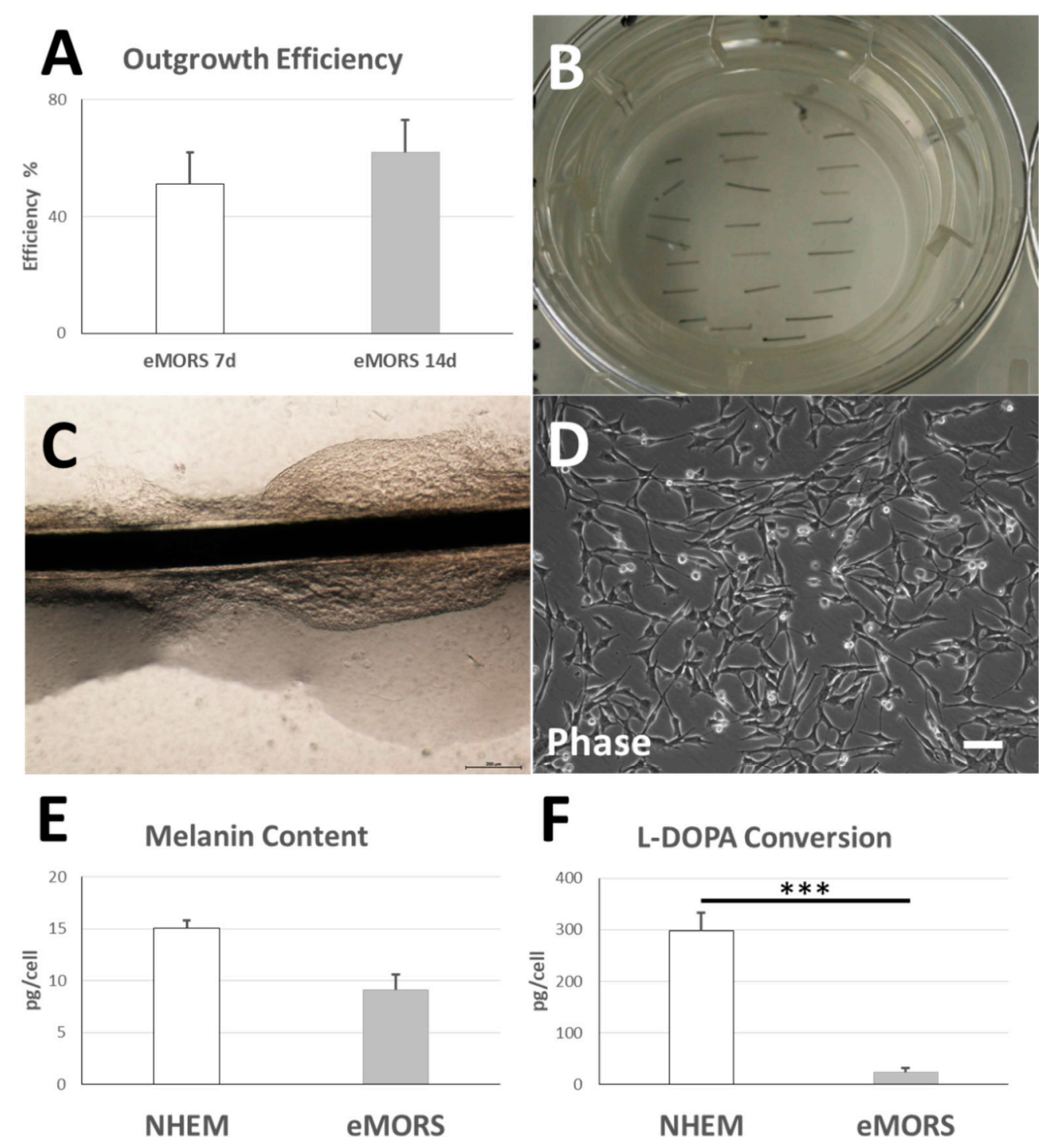

Figure 2. Isolation of horse hair follicles, migration of cells from the ORS and cell culture. Demonstration of isolating horse HM from the forelock hair follicle. The forehead skin tissue was excised from the equine forehead region, disinfected and intensively rinsed. The subcutaneous tissue was removed, and skin was chopped into long pieces. After collagenase digestion, the intact hair follicles were isolated from the loosened skin tissue. (A) Cell outgrowth efficiency of eMORS from the ORS. (B) Air-liquid interface culture on Transwell membrane. (C) ORS cells migrated out from the ORS onto the porous nylon membrane. (D) After being harvested from the membrane, horse HM adhered and proliferated on cell culture flasks in DLMS medium, displaying typical melanocyte morphology. (E) Melanin content of eMORS and L-DOPA conversion (F) compared with NHEM. Results were shown with mean \pm SD, ${ }^{* * *} p<0.001$. Magnification (D): $10 \times$, scale bar: $200 \mu \mathrm{m}$.

\subsection{Melanin Content and Enzymatic L-DOPA Conversion Activity}

eMORS exhibited melanin content of $9.13 \pm 1.45 \mu \mathrm{g} / \mathrm{mL}$ that was non-significantly different from the content of $15.07 \pm 0.72 \mu \mathrm{g} / \mathrm{mL}$ measured in NHEM controls $(p=0.093)$ (Figure 2E). The activity of tyrosinase in the lysate of eMORS resulted in producing $22.6 \pm 9 \mu \mathrm{g} / \mathrm{mL}$ melanin (Figure 2E), which was 2.48-fold higher than the melanin content in eMORS pellet $(* * *=0.0001)$ and 13.14-fold lower in comparison to the amount of $297.7 \pm 35.39 \mu \mathrm{g} / \mathrm{mL}$ produced in NHEM lysate controls $\left.{ }^{* * *} p=0.000027\right)$ (Figure $2 \mathrm{~F}$ ). 


\subsection{Subcellular Distribution of Sox2, $\alpha-S M A$, Vimentin, Tyrosinase and S100 $\beta$}

The eMORS expressed Sox2, $\alpha$-SMA, vimentin, tyrosinase and S100ß (Figure 3). Sox2 and Tyrosinase location was limited to the perinuclear region and soma (Figure $3 \mathrm{~A}, \mathrm{C}$ ). The $\alpha$-SMA and vimentin pattern was typical to that of a cytoskeleton (Figure $3 \mathrm{~B}, \mathrm{D}$ ) and it reflected the conventional polydendritic morphology of melanocytes. The pattern of S100ß was chiefly observable as punctative aggregations, distributed perinuclearlyand along the dendrites, hereby fitting the subcellular localization of melanosomes.
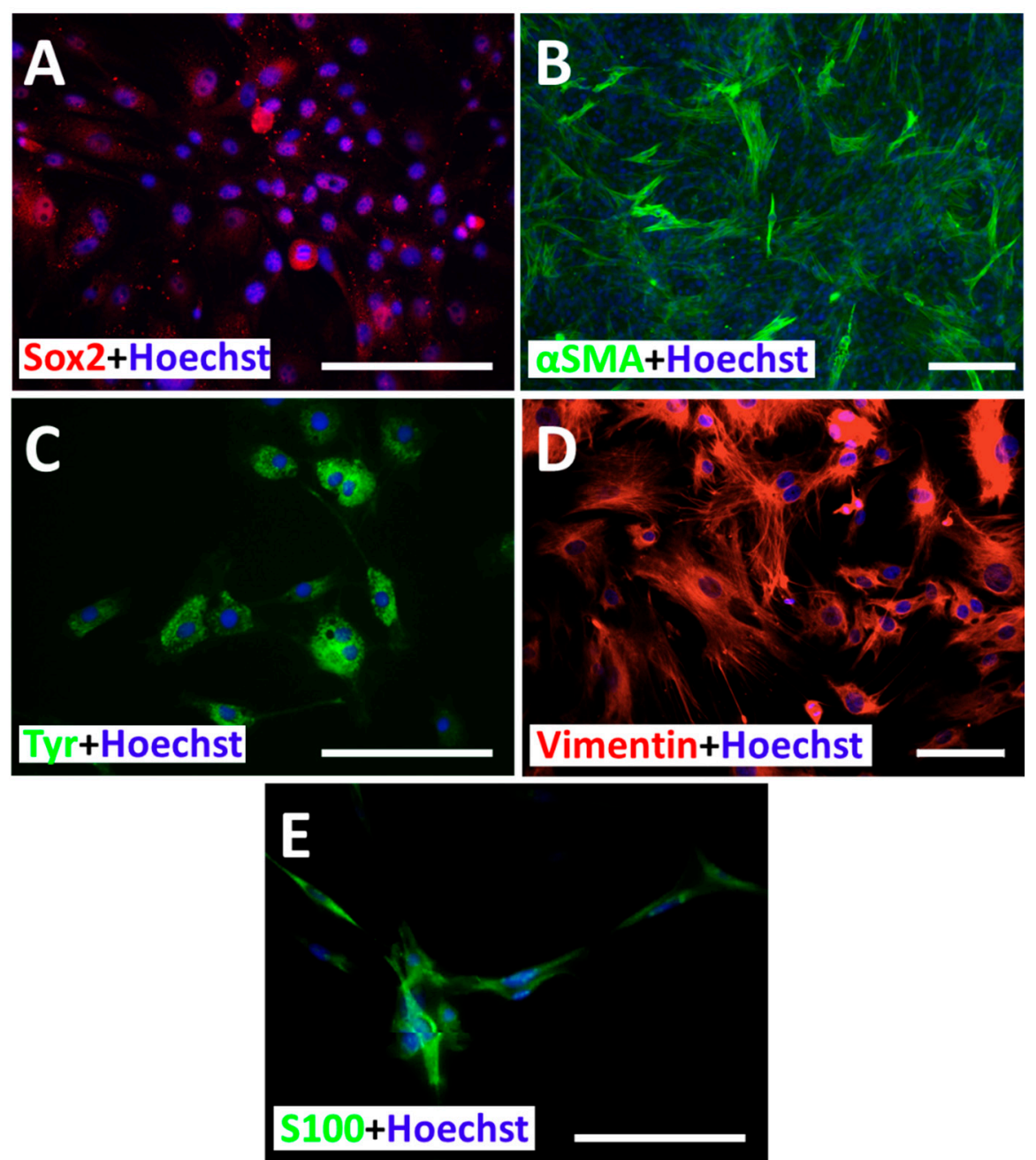

Figure 3. Horse hair follicle melanocytes in culture with immunostaining. Immunofluorescent staining of horse HM with antibodies against (A) Sox2, (B) $\alpha \mathrm{SMA}$, (C) Tyrosinase, (D) Vimentin and (E) S100ß. Nuclear counterstaining was performed using Hoechst 33342. Magnification: (A,C,D) 40×, (B) $20 \times$; scale bar: $100 \mu \mathrm{m}$.

\subsection{Gene Expressions of MITF, TYR, CKIT and PMEL}

Gene expression patterns of melanocytic markers in eMORS were assessed by the means of qRT-PCR after serial passages in the primary culture. Early passage P2 and later passage P10 were used as checkpoints. As shown in Figure 4A-D, relative gene expression of melanocyte markers MITF, TYR, CKIT and PMEL in eMORS exhibited an increasing trend at the later checkpoint. After 10 passages of cultivation in the medium DLMS, eMORS displayed non-significantly higher levels of melanocyte markers than at the early passage P2, by 5.27-fold for CKIT, 5.81-fold for TYR, 0.76-fold for MITF and 0.73-fold for PMEL $(p>0.05)$. 
A

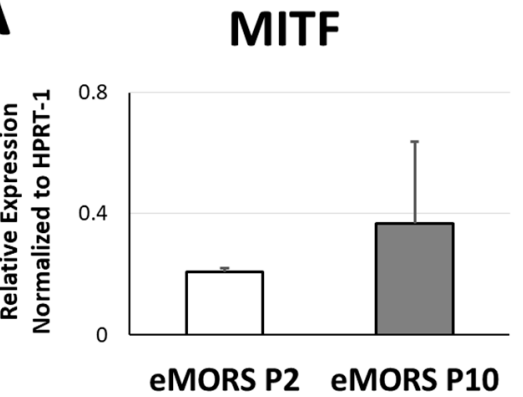

C

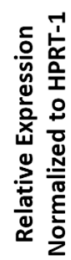

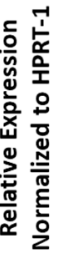

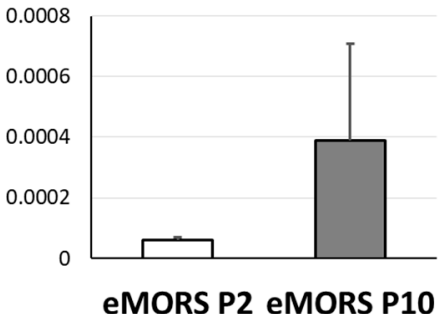

B TYR

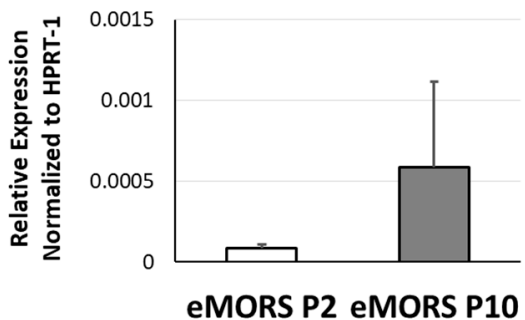

D

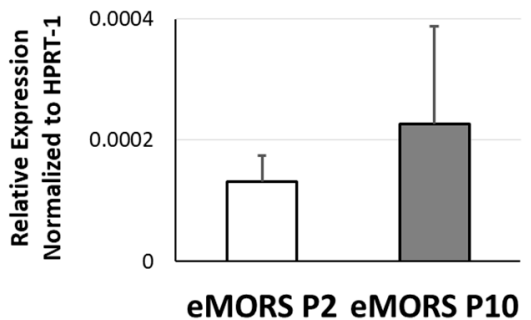

Figure 4. Gene expression of melanocyte markers in eMORS. qRT-PCR indicated gene expressions of (A) MITF, (B) TYR, (C) CKIT, (D) PMEL in eMORS at passage 2 and passage 10. Housekeeping gene HPRT-1 was used as the internal reference for each gene, and relative expressions are showed with mean \pm SD.

\section{Discussion}

This study demonstrates that the method for culturing human melanocytes from the hair follicle ORS is applicable in other species, in this case the horse. To the best of our knowledge, this is the first study to report in vitro isolation and culture of equine melanocytes altogether as well as from the hair follicle ORS.

Compared to the equivalent runs using human hair follicles, the overall intensity of cell migration from the ORS onto the nylon mesh was lower in equine. Migration of eMORS from the follicles within 7 days was at $44 \%$ and after 14 days at $62 \%$, (Figure 2A) which was less efficient than in HUMORS, previously recorded with $79 \%$ of human follicles yielding migrating HUMORS in 7 days and $90 \%$ of successful attempts in 14 days [24]. The migration progression in both methods was hereby comparable, showing an increase of $22 \%$ from day 7 to day 14 in eMORS and $14 \%$ in HUMORS, hereby demonstrating similar dynamics in human and equine.

Addition of equine serum to the culture medium was apparently a justified attempt of approximating physiological conditions within an in vitro culture. Equine serum proved to be a very important factor for a successful set-up of sustainable, passageable culture of eMORS, since its omittance from the medium formulation resulted in failing cultures. This finding is concordant to the historical data focused on cell growth of equine adrenocortical cell culture in equine serum-enriched medium [25].

The culture and selection methods earlier applied in humans yielded same type of effects in horses, resulting in pure, stable long-term culture of eMORS over the observed 12 passages. The cultured eMORS developed stellar morphology typical for melanocytes, as shown by immunofluorescence staining of cytoskeleton proteins $\alpha$-SMA and vimentin, preceded by a bi- and tri-dendritic phase in early passages, as visible in the phase-contrast photo documentation (Figures 2D and 3B,D). Aligned with the morphology, subcellular distribution of Sox2 suggests that the melanocytic developmental program was set in motion in eMORS. Sox2, an activator of microphthalmia-induced transcription factor (MITF), the central protein of melanocyte program in melanocytes, was also present in the soma as well as perinuclear in the eMORS, where the MITF activation habitually takes place. 
Distribution of S100 signals in form of melanosome-like aggregations in perinuclear region and dendrites indicates an onset of melanotic processes, i.e., production and vesicular secretion of melanin.

Cross-reactivity of antibodies against human $\alpha$-SMA, vimentin and Sox 2 towards the corresponding equine proteins have been reported earlier [26], which was also based on their high coding sequence match on the evolutionary conserved sequences (Supplementary Table S1). For those reasons, a modest immunofluorescent labelling was expected and a certain non-typical display of the subcellular distribution could be duly anticipated.

Based on the available results of the immunofluorescent labelling, the subcellular distribution of melanosome vesicles ranged from perinuclear to the soma and partially along dendrites, reflecting a pattern characteristic for melanocytes in the early developmental phase. In accordance with melanosome allocation, tyrosinase, the principal enzyme in melanogenesis and a constituent of melanosomes [27], also exhibited subcellular distribution aggregated more in the perinuclear and somatic part of the cytoplasm and less intensive in the dendrites. Concordantly, conversion of L-DOPA, which mirrors tyrosinase activity, was 13-fold lower in eMORS than that of NHEM controls. Lower conversion rates reflect lower tyrosinase activity, which is also typical of early melanogenesis. On the other hand, comparable melanin content in eMORS to that of NHEM clearly demonstrated substantial melanotic activity in eMORS. The aforementioned indicates that the eMORS were carrying out an early melanocytic program and defines them as partially melanotic melanocytes, analogue to HUMORS in the analogue explant procedure. $\mathrm{Ab}$ sence of tyrosinase expression in $\mathrm{p} 1$ (data not shown), its presence in later passages and the S100ß melanosome-like distribution indicate the unfolding of melanotic program in eMORS (Figure 3C,E).

The post mortem sampling that we utilized to optimize the method of culturing eMORS could be translated to ex vivo by performing a downsized biopsy on living animals. Theoretically, the surface of skin specimen used for the method of sampling intact follicles could be reduced to a size of $0.1 \mathrm{~cm}^{2}$, based on down-calculation from 521 follicles $/ \mathrm{cm}^{2}$ skin and aiming for a starting amount of 50 follicles, which were typically used to culture and characterize HUMORS in preceding studies in human. In practice, it is difficult to sample skin area of such miniscule size, therefore the necessary sample can be reduced to a single $1 \mathrm{~mm}$-wide $\left(0.031 \mathrm{~cm}^{2}\right)$ punch biopsy. This is easily performable in living animals, requiring local anesthesia and causing very low to none donor-site morbidity. Such swift procedure would yield over 150 follicles and it would remain by far the least invasive known method of sampling follicles, and stem cells altogether, in horse.

The method of culturing eMORS does not have a significant therapeutic output, since treating pigmentation disorders is simply not pursued in the horse veterinary practice. Although cosmetic concerns do not play an important role in daily veterinarian routine, there are implications for practice and research: tumors of melanocytic origin, the socalled white horse melanoma, present an area of intense clinical research interest. Here, tumors and melanoma cells derived from them have been characterized [28-30]. The underlying mutation has been looked into by Rosengren et al. 2008 and identified as a $4.6 \mathrm{~kb}$ duplication in Syntaxin 17 on chromosome 25. Therefore, our work presented here provides the experimental basis for a simple and quick cell culture set-up from a minimal sample allowing for further links between basic and clinical research. In addition to that, it enables further insight into horse hair follicle biology as well as comparison of follicle- and placode structures across different species. Likely, though, the method will also serve as a base for isolating other types of cells from horse hair follicles, possibly including ORS stem cells for actual regenerative therapeutic purposes.

\section{Conclusions}

This study presents a translation from the human model to equine skin, which is an efficient technique of isolating and cultivating functional melanocytes from the hair follicle ORS using non-invasive plucking and air-liquid outgrowth method. This is the 
first study reporting an isolation and culture of equine melanocytes. The cultured eMORS displayed normal morphological and functional features compared with human normal epidermal melanocytes.

Supplementary Materials: The following are available online at https:/ /www.mdpi.com/2227-9 717/9/1/177/s1, Table S1: Coding sequence homogeneity between human and equine tyrosinase, Sox2, vimentin, alpha-smooth muscle actin and S100ß.

Author Contributions: Conceptualization, V.S. and S.H.; formal analysis, H.L. and R.Z.; funding acquisition, J.-C.S., V.S. and B.L.; investigation, H.L. and J.K.M.; methodology, J.K.M.; supervision, V.S. and B.L.; visualization, H.L. and J.K.M.; writing — original draft, H.L., V.S. and A.K.S.; writingreview and editing, A.B., S.G. and B.L. All authors have read and agreed to the published version of the manuscript.

Funding: This research was supported by the Department of Cranial Maxillofacial Plastic Surgery, University Clinic Leipzig, Germany, Saxon Ministry of Science and Fine Arts (SMWK) to Saxon Incubator for Clinical Translation (SIKT) to vs. and German Research Council (DFG) SFB TRR 67 B12 to vs. and B3 to SF and JCS. We gratefully acknowledge support from Leipzig University for Open Access Publishing.

Institutional Review Board Statement: The study was conducted according to the guidelines of the Declaration of Helsinki and usage of human samples were approved by the Ethical Committee of Medical Faculty, University of Leipzig (427/16-ek, approved in 27.02.2017).

Informed Consent Statement: Not applicable.

Data Availability Statement: The data presented in this study are available on request from the corresponding author.

Acknowledgments: We are grateful to Tina Kottek, Katrin Rabe, Sabine Hecht and Heike Konow. We gratefully acknowledge support from Leipzig University for Open Access Publishing.

Conflicts of Interest: The authors declare no conflict of interest.

\section{References}

1. Cotsarelis, G.; Sun, T.T.; Lavker, R.M. Label-retaining cells reside in the bulge area of pilosebaceous unit: Implications for follicular stem cells, hair cycle, and skin carcinogenesis. Cell 1990, 61, 1329-1337. [CrossRef]

2. Ma, H.-J.; Yue, X.-Z.; Wang, D.-G.; Li, C.-R.; Zhu, W.-Y. A modified method for purifying amelanotic melanocytes from human hair follicles. J. Dermatol. 2006, 33, 239-248. [CrossRef]

3. Hoffman, R.M. The pluripotency of hair follicle stem cells. Cell Cycle 2006, 5, 232-233. [CrossRef] [PubMed]

4. Tobin, D.J.; Swanson, N.; Pittelkow, M.; Peters, E.; Schallreuter, K. Melanocytes are not absent in lesional skin of long duration vitiligo. J. Pathol. 2000, 191, 407-416. [CrossRef]

5. Na, G.Y.; Paek, S.H.; Park, B.C.; Kim, D.W.; Lee, W.J.; Lee, S.J.; Kim, M.K.; Kim, J.C. Isolation and characterization of outer root sheath melanocytes of human hair follicles. Br. J. Dermatol. 2006, 155, 902-909. [CrossRef]

6. Zhu, W.Y.; Zhang, R.Z.; Ma, H.J.; Wang, D.G. Isolation and culture of amelanotic melanocytes from human hair follicles. Pigment. Cell Res. 2004, 17, 668-673. [CrossRef] [PubMed]

7. Kauser, S.B.; Thody, A.J.; Schallreuter, K.U.; Gummer, C.L.; Tobin, D.J. A Fully Functional Proopiomelanocortin/Melanocortin-1 Receptor System Regulates the Differentiation of Human Scalp Hair Follicle Melanocytes. Endocrinology 2005, 146, 532-543. [CrossRef]

8. Kauser, S.; Thody, A.J.; Schallreuter, K.U.; Gummer, C.L.; Tobin, D.J. $\beta$-Endorphin as a Regulator of Human Hair Follicle Melanocyte Biology. J. Investig. Dermatol. 2004, 123, 184-195. [CrossRef]

9. Limat, A.; French, L.E.; Blal, L.; Saurat, J.H.; Hunziker, T.; Salomon, D. Organotypic cultures of autologous hair follicle keratinocytes for the treatment of recurrent leg ulcers. J. Am. Acad. Dermatol. 2003, 48, 207-214. [CrossRef]

10. Aasen, T.; Belmonte, J.C.I. Isolation and cultivation of human keratinocytes from skin or plucked hair for the generation of induced pluripotent stem cells. Nat. Protoc. 2010, 5, 371. [CrossRef]

11. Seo, Y.-K.; Lee, D.-H.; Shin, Y.-H.; You, B.-Y.; Lee, K.-M.; Song, K.-Y.; Seo, S.-J.; Whang, S.-J.; Kim, Y.-J.; Yang, E.-K. Development of isolation and cultivation method for outer root sheath cells from human hair follicle and construction of bioartificial skin. $J$. Biotechnol. Bioprocess. Eng. 2003, 8, 151-157. [CrossRef]

12. Nishimura, E.K. Melanocyte stem cells: A melanocyte reservoir in hair follicles for hair and skin pigmentation. Pigment. Cell Melanoma Res. 2011, 24, 401-410. [CrossRef] [PubMed]

13. Wang, D.; Xu, X.; Ma, H.; Yue, X.; Li, C.; Zhu, W. Optimization of the method for the culture of melanocyte precursors from hair follicles and their activation by 1, 25-dihydroxyvitamin D3. J. Exp. Therapeutic Med. 2013, 6, 967-972. [CrossRef] [PubMed] 
14. Dieckmann, C.; Milkova, L.; Hunziker, T.; Emmendörffer, A.; Simon, J.C. Human melanocytes can be isolated, propagated and expanded from plucked anagen hair follicles. J. Exp. Dermatol. 2010, 19, 543-545. [CrossRef] [PubMed]

15. Savkovic, V.; Dieckmann, C.; Milkova, L.; Simon, J.C. Improved method of differentiation, selection and amplification of human melanocytes from the hair follicle cell pool. Exp. Dermatol. 2012, 21, 948-950. [CrossRef]

16. Savkovic, V.; Dieckmann, C.; Simon, J.-C.; Schulz-Siegmund, M.; Hacker, M. Method for Deriving Melanocytes from the Hair Follicle Outer Root Sheath and Preparation for Grafting. World Patent WO2013060899A2, 2 May 2013.

17. Marie, S.; Christina, D.; Katrin, R.; Simon, J.-C.; Vuk, S. Differentiating the Stem Cell Pool of Human Hair Follicle Outer Root Sheath into Functional Melanocytes. Stem Cells Tissue Repair 2014, 1210, 203-227.

18. Korzynska, A.; Zychowicz, M. A Method of Estimation of the Cell Doubling Time on Basis of the Cell Culture Monitoring Data. Biocybern. Biomed. Eng. 2008, 28, 75-82.

19. Cimadamore, F.; Shah, M.; Amador-Arjona, A.; Navarro-Peran, E.; Chen, C.; Huang, C.-T.; Terskikh, A.V. SOX2 modulates levels of MITF in normal human melanocytes, and melanoma lines in vitro. Pigment. Cell Melanoma Res. 2012, 25, 533-536. [CrossRef]

20. El-Koraie, A.F.; Baddour, N.M.; Adam, A.G.; El-Kashef, E.H.; El Nahas, A.M. Cytoskeletal protein expression and regenerative markers in schistosomal nephropathy. Nephrol. Dial. Transplant. 2002, 17, 803-812. [CrossRef]

21. Harding, P.; Moosajee, M. The Molecular Basis of Human Anophthalmia and Microphthalmia. J. Dev. Biol. 2019, 7, 16. [CrossRef]

22. Shibahara, S.; Takeda, K.; Yasumoto, K.-I.; Udono, T.; Watanabe, K.-I.; Saito, H.; Takahashi, K. Microphthalmia-Associated Transcription Factor (MITF): Multiplicity in Structure, Function, and Regulation. J. Investig. Dermatol. Symp. Proc. 2001, 6, 99-104. [CrossRef] [PubMed]

23. Garcia-Jimenez, A.; Teruel-Puche, J.A.; Garcia-Ruiz, P.A.; Berna, J.; Rodríguez-López, J.N.; Tudela, J.; Garcia-Canovas, F. Action of 2,2',4,4'-tetrahydroxybenzophenone in the biosynthesis pathway of melanin. Int. J. Biol. Macromol. 2017, 98, 622-629. [CrossRef] [PubMed]

24. Sülflow, K.; Schneider, M.; Loth, T.; Kascholke, C.; Schulz-Siegmund, M.; Hacker, M.C.; Simon, J.C.; Savkovic, V. Melanocytes from the outer root sheath of human hair and epidermal melanocytes display improved melanotic features in the niche provided by cGEL, oligomer-cross-linked gelatin-based hydrogel. J. Biomed. Mater. Res. Part A 2016, 104, 3115-3126. [CrossRef] [PubMed]

25. Hornsby, P.J.; Sturek, M.; Harris, S.E.; Simonian, M.H. Serum and growth factor requirements for proliferation of human adrenocortical cells in culture: Comparison with bovine adrenocortical cells. Vitro 1983, 19, 863-869. [CrossRef] [PubMed]

26. Michler, J.K.; Hillmann, A.; Savkovic, V.; Mülling, C.K.W. Horse hair follicles: A novel dermal stem cell source for equine regenerative medicine. Cytom. Part A 2018, 93, 104-114. [CrossRef]

27. Wasmeier, C.; Hume, A.N.; Bolasco, G.; Seabra, M.C. Melanosomes at a glance. J. Cell Sci. 2008, 121, 3995-3999. [CrossRef]

28. Chapman, S.W.; Metzger, N.; Grest, P.; Feige, K.; von Rechenberg, B.; Auer, J.A.; Hottiger, M.O. Isolation, establishment, and characterization of ex vivo equine melanoma cell cultures. In Vitro Cell. Dev. Biol. Anim. 2009, 45, 152-162. [CrossRef]

29. Seltenhammer, M.H.; Simhofer, H.; Scherzer, S.; Zechner, P.; Curik, I.; Sölkner, J.; Brandt, S.M.; Jansen, B.; Pehamberger, H.; Eisenmenger, E. Equine melanoma in a population of 296 grey Lipizzaner horses. Equine Vet. J. 2003, 35, 153-157. [CrossRef]

30. Phillips, J.C.; Lembcke, L.M. Equine melanocytic tumors. Vet. Clin. N. Am. Equine Pract. 2013, 29, 673-687. [CrossRef] 